\title{
Proposta metodológica para elaboração de um indicador de fragilidade ambiental para fragmentos florestais
}

\section{Methodological proposal for elaboration of an environmental fragility indicator for forest fragments}

\author{
Bruno Yuji Takikawa*1 $₫\left(\mathbb{D}\right.$, Darllan Collins da Cunha e Silva ${ }^{2} \bowtie(\mathbb{D}$, \\ Roberto Wagner Lourenço ${ }^{1} \bowtie(1)$ \\ ${ }^{1}$ Instituto de Ciência e Tecnologia, Universidade Estadual Paulista Júlio de Mesquita Filho, \\ Sorocaba, São Paulo, Brasil \\ ${ }^{2}$ Cursos de Engenharia Agronômica e Engenharia de Pesca, Universidade Estadual Paulista Júlio \\ de Mesquita Filho, Registro, São Paulo, Brasil \\ E-mail: darllan.collins@unesp.br (DCCS); roberto.lourenco@unesp.br (RWL) \\ *E-mail para correspondência: bruno.takikawa@gmail.com
}

\author{
Recebido (Received): 02/06/2020 \\ Aceito (Accepted): 11/10/2020
}

\begin{abstract}
Resumo: A expansão das atividades antrópicas resultou na perda de quase $88 \%$ da cobertura florestal original da Mata Atlântica, impulsionando o processo de fragmentação e a perda de habitat, principais causas de extinção da fauna e flora. Diante disso, este artigo teve por objetivo desenvolver uma metodologia para identificação de fragmentos florestais prioritários para ações de proteção ambiental em uma bacia hidrográfica com forte ação antrópica. O indicador elaborado teve como escopo a avaliação dos parâmetros relacionados à forma, uso de entorno do fragmento e a qualidade da saúde da vegetação. Para tanto, foi gerado o Índice de Circularidade (IC), o Índice de Efeito de Borda (IEB) e o Índice de Qualidade Biofísica do Fragmento (IQBF). Foi aplicado o método de Análise Hierárquica de Processos (AHP) para atribuir pesos relacionados à importância de cada um dos índices resultando na elaboração de um indicador que pudesse demonstrar o estado de fragilidade das florestas da área de estudo, chamado de Indicador de Fragilidade Florestal (IFF). Os resultados mostraram que dos 27 fragmentos florestais estudados, apenas 01 fragmento foi considerado com baixa fragilidade, $22 \mathrm{com}$ média fragilidade e $04 \mathrm{com}$ alta fragilidade florestal. Pode-se afirmar que os resultados do IFF mostraram que o tipo de uso das terras adjacentes aos fragmentos florestais é o principal fator em relação aos demais. Finalmente, a modelagem utilizando dados espaciais e AHP se mostraram bastante úteis e ágeis na determinação de áreas prioritárias para gestão e conservação de remanescentes florestais, podendo ser replicada para outras bacias hidrográficas.
\end{abstract}

Palavras-chaves: Geoprocessamento; Mata Atlântica; AHP.

Abstract: The expansion of human activities resulted in the loss of almost $88 \%$ of the original forest cover of the Atlantic Forest, driving the fragmentation process and the loss of habitat, the main causes of fauna and flora extinction. Therefore, this article aimed to develop a methodology for identifying priority forest fragments for environmental protection actions in a hydrographic basin with strong anthropic action. The indicator elaborated had as scope an evaluation of the parameters related to the shape, use of fragment and quality of vegetation health. For this, the Circularity Index (CI), the Edge Effect Index (EEI) and the Fragment Biophysical Quality Index (FBQI) were created. From the integration of these three indices, the Forest Fragility Indicator (FFI) was created. The Hierarchical Process Analysis (HPA) method was applied to assign weights related to the importance of each index in the IFF construction process. The results obtained were 27 forest fragments studied, only 01 fragment was considered with low fragility, 22 with medium fragility and 04 with high forest fragility. It is possible to indicate which FFI results show the type of land use adjacent to the forest fragments or the main factor in relation to the others. Finally, a model that uses spatial data and HPA can be quite useful and determine priority areas for the management and conservation of forest remnants, which can be replicated to other river basins.

Keywords: Geoprocessing; Atlantic Forest; HPA. 


\section{Introdução}

A Mata Atlântica é um dos biomas de maior diversidade do planeta. Entretanto, a exploração de recursos naturais, as atividades agropastoris e a aglomeração urbana e industrial causaram a sua fragmentação e descaracterização, restando aproximadamente $10 \%$ de sua cobertura original. (KRONKA et al., 2005; RIBEIRO et al., 2009). Mesmo perdendo a maior parte de sua cobertura, a Mata Atlântica apresenta um alto índice de endemismo de aves, mamíferos, répteis, anfíbios e plantas vasculares (RIBEIRO et al., 2009; SILVA et al., 2018). A grande perda de habitat aliada à alta biodiversidade faz com que o ambiente seja apontado como um hotspot mundial (LAURANCE, 2009).

A fragmentação florestal, além de provocar perda de área, pode modificar o seu equilíbrio ecodinâmico, causando alterações das condições ambientais de temperatura, umidade e radiação solar, o que pode resultar na diminuição da abundância e riqueza das espécies, na variabilidade genética e nos padrões de dispersão, reprodução e predação (FAHRIG, 2003; BORGES et al., 2004).

Além disso, o isolamento dos fragmentos também é influenciado pelo tipo de vizinhança, uma vez que o uso da terra do entorno pode causar um efeito de borda provocando desequilíbrio em relação ao fluxo das espécies. Como exemplo, pode-se citar a baixa permeabilidade entre os fragmentos, a qual se relaciona com a alta probabilidade de extinção local e baixa chance de recolonização de espécies. (HANSKI; GILPIN, 1997; AMORIM et al., 2019).

A evolução das geotecnologias tem auxiliado em diversas áreas, como gestão pública, saúde, setor florestal e análises ambientais. Atividades que antes necessitavam de alta demanda de mão de obra e altos custos financeiros passaram a ser executadas em um tempo mais curto e com melhores resultados, sendo bastante útil para gestão ambiental (BURROUGH, 1998; SANTOS et al., 2012).

Vários trabalhos utilizaram geoprocessamento para análise de fragmentos florestais. Lourenço et al. (2014) e Lourenço et al. (2015) utilizaram técnicas de análise espacial e estatística para a elaboração de indicadores de sustentabilidade florestal, obtendo resultados satisfatórios para gestão e preservação de florestas em bacias hidrográficas. Sales et al. (2017) desenvolveram uma metodologia com base em levantamento de avifauna e geoprocessamento para avaliar os riscos à biodiversidade em fragmentos florestais com intervenção antrópica.

Diante do exposto, o objetivo deste trabalho foi demonstrar uma metodologia para a construção de um Indicador de Fragilidade Florestal (IFF) que busca identificar fragmentos florestais prioritários para ações de proteção ambiental em bacias hidrográficas.

\section{Materiais e métodos}

\section{1. Área de estudo}

O presente estudo foi realizado na Bacia Hidrográfica do rio Una (Figura 1), a qual está inserida inteiramente no município de Ibiúna do Estado de São Paulo. A bacia possui 9.816 hectares e seu principal rio é o Rio Una. Possui uma extensão de $25 \mathrm{~km}$, e juntamente com outros rios apresenta relevante importância para produção de energia, bem como o abastecimento para boa parte da população da região (SALES et al., 2019).

O clima predominante da região é do tipo $\mathrm{Cwb}$, com verão chuvoso e inverno com estiagem, segundo a classificação de Köeppen, enquanto, a vegetação original é a ombrófila densa montana (SILVA et al., 2016). Nesta bacia há predominância de atividades de reflorestamento, pastagem e lavouras permanentes e temporárias (SILVA et al., 2016). 


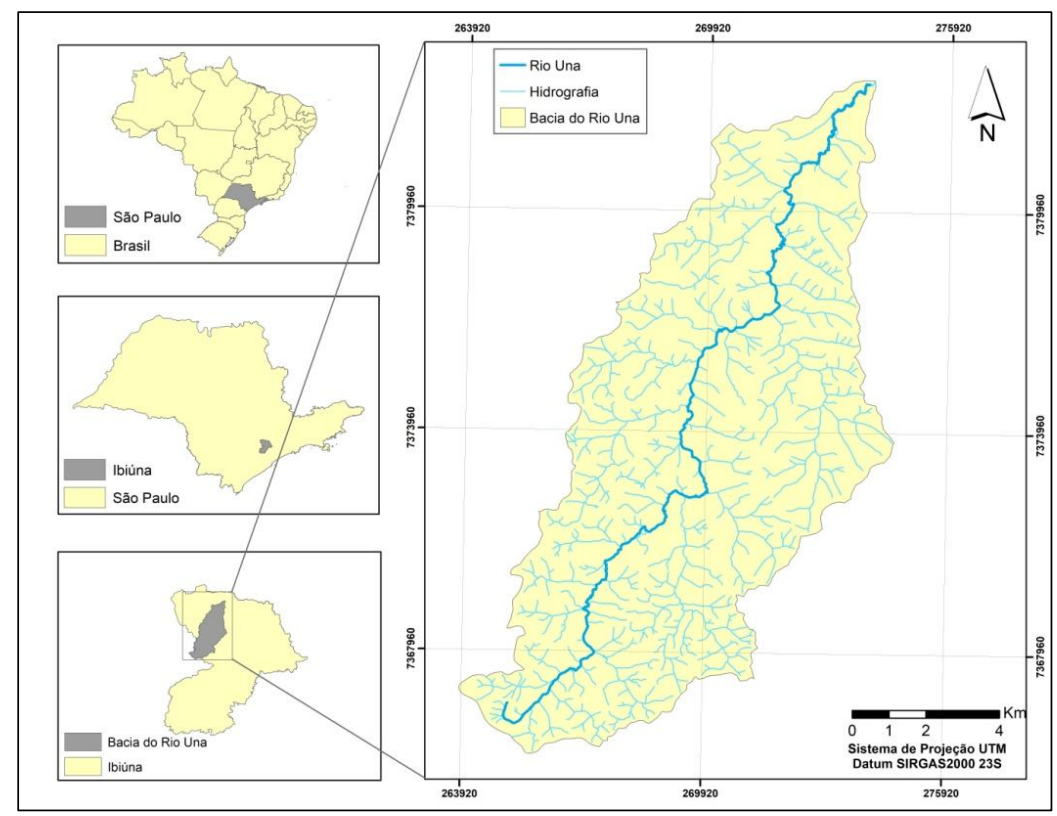

Figura 1: Bacia Hidrográfica do Rio Una, SP, Brasil.

\subsection{Mapeamento dos fragmentos florestais}

O Mapa de Uso da Terra foi elaborado através da interpretação visual de imagens do satélite RapidEye com resolução espacial de 5,0 metros e período de referência de agosto/2014, e posteriormente retificada com o uso das imagens disponíveis do Google Earth Pro e observações em campo para o ano de 2018. As classes foram divididas em Florestas, Área degradada, Lâmina d'água, Área urbana, Área rural, Silvicultura (cultura permanente), Cultura temporária, Pastagem, Aterro sanitário, adaptado do Manual Técnico de Uso da Terra (IBGE, 2013a).

Em seguida, as unidades amostrais da categoria Florestas foram separadas (Figura 2A), e a partir disso, foi realizado um procedimento de identificação das áreas área core dos fragmentos (OLIVEIRA et al., 2015), considerando-se uma faixa com distância de 50 metros da borda para o interior do fragmento para eliminando o efeito de borda (Figura 2B). Estas áreas core foram individualizadas e os fragmentos com área central maior que 10 hectares foram selecionados (Figura 2C), e sobre eles foi construído um buffer em seu perímetro de 50 metros, constituindo-se as novas áreas de estudo (Figura 2D).

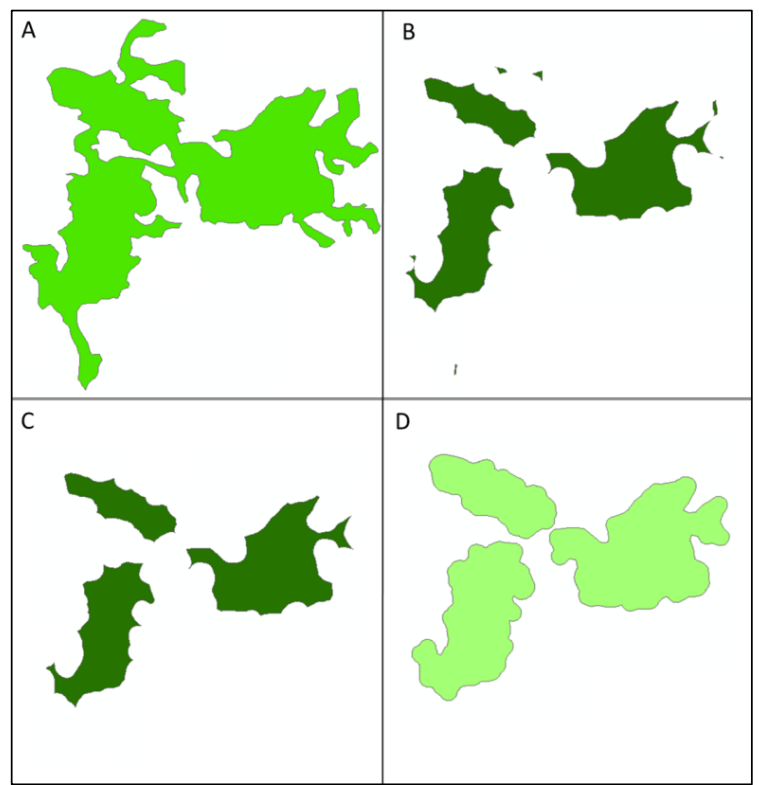

Figura 2A-D: Seleção e remodelagem dos fragmentos florestais. Legenda: A: Fragmento florestal. B: Áreas centrais. C: Exclusão dos fragmentos com área central menor que 10ha. D: Novos polígonos delimitados. 


\subsection{Elaboração do indicador de fragilidade florestal (IFF)}

O IFF foi gerado a partir da integração do Índice de Circularidade (IC), Índice de Efeito de Borda (IEB) e Índice de Qualidade Biofísica do Fragmento (IQBF) obtido dos fragmentos identificados no item 2.2.

O IC avalia a geometria dos fragmentos florestais por meio da relação perímetro-área. Quanto menor a relação perímetro-área, maior a proporção de área central, sendo assim adequados para a preservação por possuírem mais áreas livres do efeito de borda (LAURANCE e VASCONCELOS, 2009). O IC foi calculado através da Equação 1.

$$
I C=2 \frac{\sqrt{\pi * A}}{P}
$$

Sendo: $\mathrm{IC}=$ Índice de Circularidade; A = Área do fragmento florestal; P = Perímetro do fragmento florestal.

Os valores de IC variam entre 0 e 1 , sendo que quanto mais próximo de 1 maior é a proporção de área central, normalmente com tendência de ser arredondado e, quanto mais distante de 1, mais alongada e consequentemente menor a proporção de área central, com maior susceptibilidade aos efeitos de borda e possibilidade de fragmentação. Os resultados obtidos foram classificados conforme Viana e Pinheiro (1998), onde índices menores que 0,6 foram considerados como muito alongados, entre 0,6 e 0,8 como alongados e acima de 0,8 arredondados.

O IEB demonstra a fragilidade dos fragmentos florestais em relação ao efeito que o uso e manejo da terra de seu entorno pode causar em relação à estabilidade biológica, pois estes podem exercer uma pressão nas bordas, dificultando o seu desenvolvimento (SALES, 2017).

O IEB neste trabalho foi adaptado de Lourenço, Silva e Sales (2014), sendo diferente pelo fato de analisar toda a área de influência dos 50 metros do fragmento florestal (Figura 3B), e não somente a linha do perímetro em contato com a borda dos fragmentos (Figura 3A).

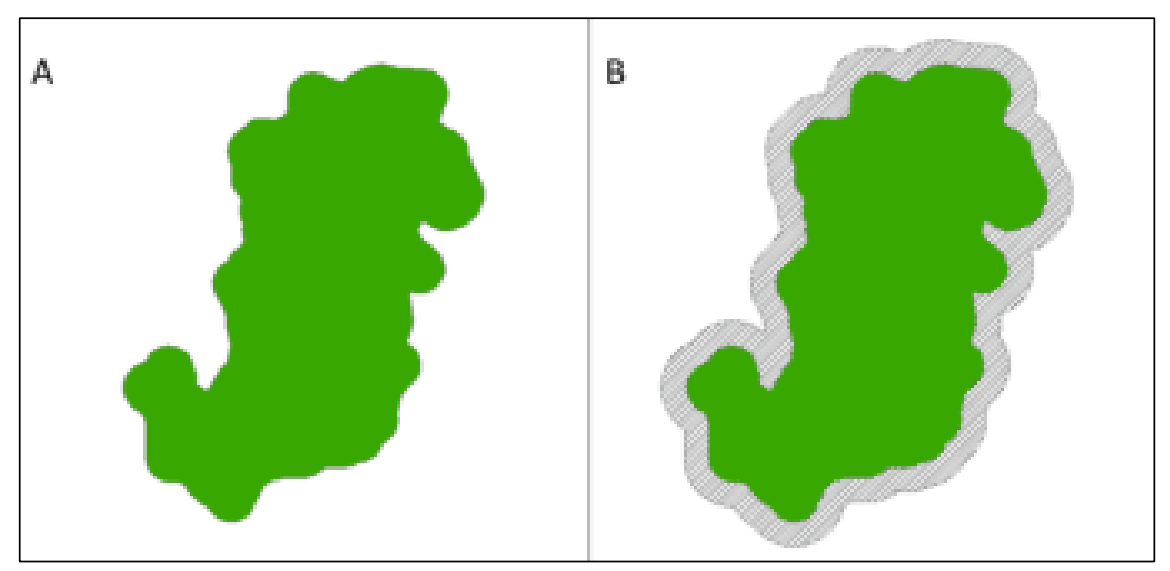

Figura 3A-B: Definição da área de influência do entorno do fragmento. Legenda: A: Fragmento florestal estudado; B: Em hachura, área do entorno do fragmento analisada.

Aos usos da terra das áreas de influência dos fragmentos foram atribuídos pesos de acordo com o grau de importância e o impacto negativo sobre os fragmentos florestais, sendo que quanto menor o peso, maior o impacto negativo exercido ao fragmento (Tabela 1).

Em seguida foi realizada a ponderação das categorias de uso da terra considerando seus respectivos pesos (Equação 2).

$$
I E B=\sum_{i=1}^{n} \frac{U_{i} x A_{i}}{A}
$$

Sendo: IEB = Índice de Efeito de Borda; $\mathrm{U}_{\mathrm{i}}=$ Peso atribuído à categoria de uso da terra; $\mathrm{A}_{\mathrm{i}}=$ Área da categoria do uso da terra; $\mathrm{A}=$ Área total do entorno. 
Tabela 1: Pesos atribuídos às categorias de uso e ocupação da terra

\begin{tabular}{lc}
\hline Categoria de uso & Peso atribuído \\
\hline Florestas & 1 \\
Corpo hídrico & 1 \\
Área degradada & 0,8 \\
Silvicultura & 0,75 \\
Pastagem & 0,7 \\
Área rural & 0,5 \\
Cultura temporária & 0,25 \\
Área urbana & 0,1 \\
Aterro sanitário & 0 \\
\hline
\end{tabular}

Fonte: Adaptado de Lourenço, Silva e Sales (2014), Sales et al. (2017) e Amorim, Souza e Lourenço (2019).

$\mathrm{O}$ índice foi elaborado para variar de 0 a 1 , sendo que valores maiores indicam menor impacto, enquanto valores próximos à 0 indicam maior impacto aos fragmentos florestais decorrentes do uso e manejo da terra. Os valores de IEB foram classificados em alto, médio e baixo impacto, sendo que valores abaixo de 0,4 referem-se à alto impacto; valores entre 0,4 e 0,7 à médio impacto e acima de 0,7 à baixo impacto em função do uso e manejo da terra nas áreas de borda dos fragmentos florestais.

O IQBF foi elaborado utilizando-se dados de teor de argila presente no solo e os valores médios do Índice de Vegetação Ajustado ao Solo (SAVI) dos fragmentos florestais. A argila possui alta retenção hídrica e facilita a atividade microbiana, fazendo com que solos argilosos apresentem maiores concentrações de componentes químicos oriundos da degradação de matéria orgânica, o que resulta em solos mais férteis, conferindo condições mais propícias para o desenvolvimento da vegetação (BITTAR, FERREIRA e CORREAA, 2013; SIMONETTI et al., 2019). O SAVI é um índice de vegetação que utiliza as propriedades espectrais do alvo, relacionando o vigor vegetativo e atributos biofísicos, e minimiza os efeitos do solo através de um fator de ajuste (HUETE, 1988; SANTOS et al., 2019).

Os dados relativos à argila foram obtidos de uma amostragem em 35 pontos realizada por Souza et al. (2018). Esses autores geraram um mapa por meio da interpolação dos valores de concentração de argila utilizando a técnica do inverso do quadrado da distância (IQD). Descrição da obtenção deste mapa pode ser encontrado em SOUZA et al., 2019 e TRINDADE et al., 2019.

O Índice de Vegetação Ajustado ao Solo - SAVI (Soil Adjusted Vegetation Index) foi obtido através da interação entre as bandas do vermelho (RED) e do infravermelho próximo (NIR) das imagens do satélite Landsat 8 de março de 2019 com resolução espacial de 30 metros. Seus valores podem variar de $-1,5$ a 1,5, sendo que quanto maior o valor, maior a proporção de vegetação. O índice foi calculado atra vés da Equação 3 .

$$
S A V I=\frac{(1+L)(N I R-R E D)}{L+N I R+R E D}
$$

Sendo: $\mathrm{L}=$ fator de ajuste com o substrato do dossel; NIR = banda do sensor referente ao infravermelho próximo; RED = banda do sensor referente ao vermelho.

Neste trabalho foi utilizado o fator de ajuste com o substrato do dossel de 0,5 , tendo em vista que a área é predominantemente de áreas florestadas compostas por matas com médias densidades de vegetação (PONZONI e SHIMABUKURO, 2007; SILVA et al., 2018). Em seguida, os valores de argila e SAVI foram normalizados para a escala de 0 a 1 e o IQBF foi calculado através da Equação 4.

$$
I Q B F=\frac{\text { teor de argila médio+SAVI médio }}{2}
$$

Finalmente, o IFF foi obtido atribuindo-se pesos para o IC, IEB e IQBF através do método AHP (SAATY, 1980). A AHP consiste em determinar pesos para os critérios a partir da comparação pareada entre eles, atribuindo prioridades em cada comparação a critério do especialista (GOMES, 2009; COSTA e BELDERRAIN, 2009; OSTOVARI et al., 2019). 
Os pesos foram validados através do cálculo do Índice de Consistência (CI) e da Razão de Consistência (RC), os quais devem ser menores que 10\% (SAATY, 1991; SILVA et al., 2017). A Equação 5 e organograma da Figura 4 demonstram os passos executados.

$$
I F F=\sum_{i=1}^{n} V_{i} \cdot P_{i}
$$

Sendo: $\mathrm{V}_{\mathrm{i}}=$ valor de cada índice; $\mathrm{P}_{\mathrm{i}}=$ peso atribuído a cada índice, conforme obtido no método AHP.

Os valores obtidos podem variar entre 0 e 1 , sendo que os maiores valores indicam os fragmentos com maior fragilidade e, portanto, os mais prioritários para ações de preservação e conservação.



Figura 4: Organograma do IFF.

\section{Resultados e discussão}

\subsection{Determinação dos fragmentos florestais avaliados}

O Mapa de Uso da Terra contendo as classes de uso da terra é apresentado na Figura 5. A partir deste mapeamento é possível observar nove classes de uso da terra, sendo que as classes de cultura temporária, pastagem e silvicultura somadas representam 50,88\% da área bacia; seguida da área de área florestal $(38,10 \%)$; campos sujos $(6,61 \%)$; área urbanizada $(3,74 \%)$ e água $(0,68 \%)$. A maior prevalência de áreas antrópicas e o menor percentual de áreas florestais podem ser explicados pelo setor econômico do município apresentar forte vocação agrícola (SILVA et al. 2017). Esta informação está amparada pelos dados de produção agrícola municipal divulgados pelo IBGE (2013b) onde São Paulo contribui com 16\% do total de produção agrícola nacional, sendo que o município de Ibiúna ocupa a $9^{a}$ posição no ranking estadual do PIB agrícola.

O aumento da produção agrícola foi intenso ao longo dos anos, conferindo uma maior taxa de supressão da vegetação e aumento da fragmentação florestal. Essas percepções também foram observadas por Sales et al. (2017) que além de identificar o maior percentual de área agrícola nesta bacia, alertou sobre as problemáticas causadas à avifauna da região devido essa fragmentação florestal.

O processo de seleção e remodelagem dos fragmentos florestais resultou em 27 fragmentos (Figura 6). Destes fragmentos, o que apresentou a menor área central foi o 12 , localizado na região central da bacia (10,41 ha). A maior área central foi encontrada no fragmento 2 na região norte da bacia $(87,40$ ha). Este fragmento está próximo a área urbanizada e, por isso, apresenta uma área maior já que a expansão territorial da área urbanizada é menor que a da agrícola na região central (SALES et al., 2017), justificando assim o número menor de fragmentos na região central da bacia. 


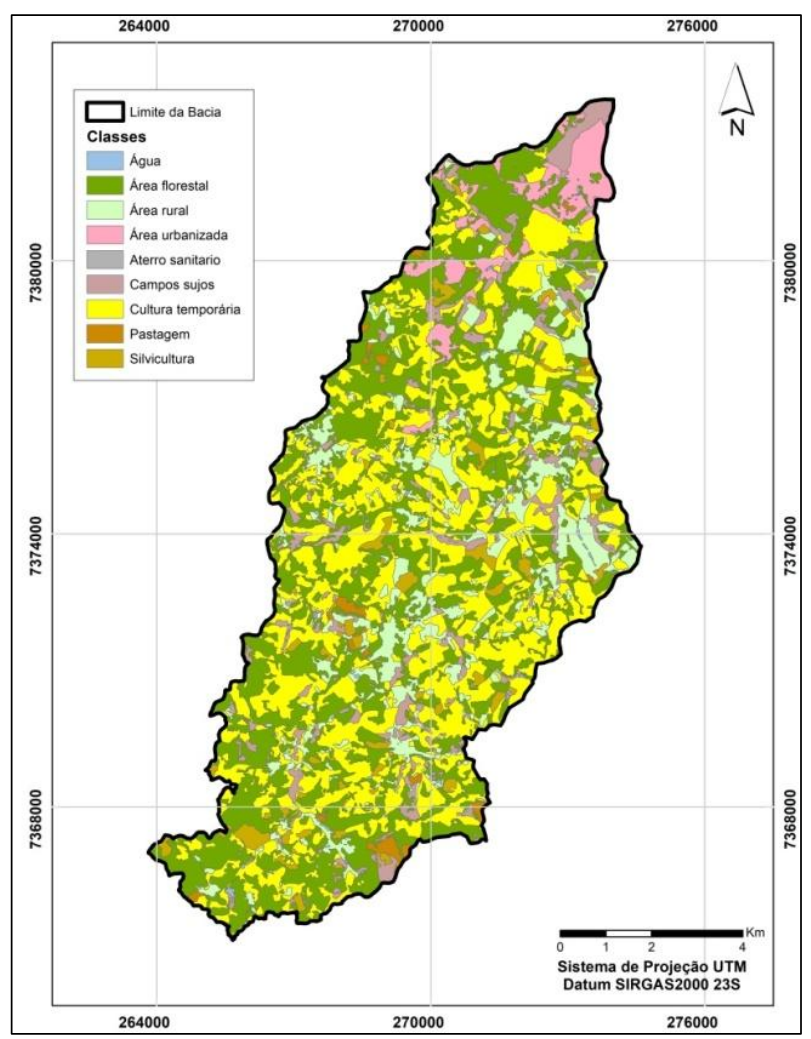

Figura 5: Mapa de Uso da Terra da Bacia Hidrográfica do rio Una, SP, Brasil.

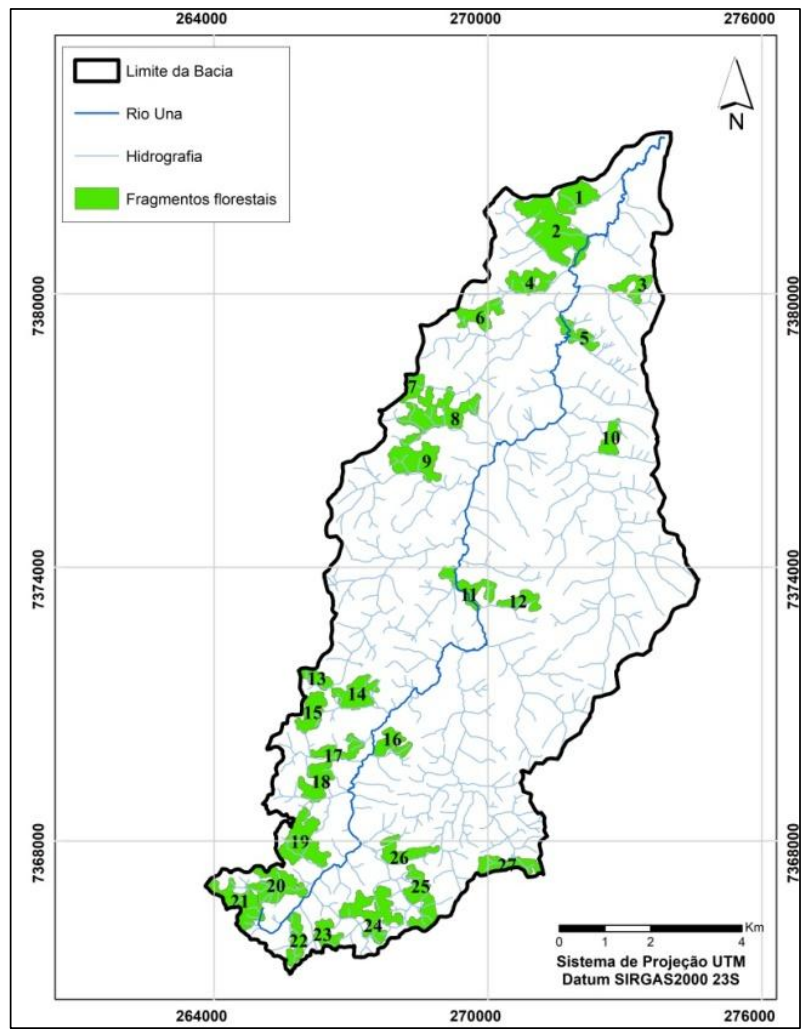

Figura 6: Fragmentos florestais estudados. Bacia Hidrográfica do rio Una, SP, Brasil.

O maior número de fragmentos ao sul da bacia deve-se a topografia acidentada que dificulta a ocupação da área por culturas agrícolas e, também, devido à baixa densidade demográfica que impõe uma pressão antrópica menor que na região central e norte da bacia (SALES et al., 2017; SALES et al., 2019).

Para 10 dos 27 fragmentos, a área do efeito de borda é maior que a área central, sendo que para o fragmento 3, parte nordeste do mapa, mostrou a maior área de efeito de borda sobre a área total do fragmento 
(61,9\%). A relação média da área central por área total do fragmento foi de $55,9 \%$, o que significa que $44,1 \%$ são referentes à área de efeito de borda. O gráfico da Figura 7 exemplifica essas relações.

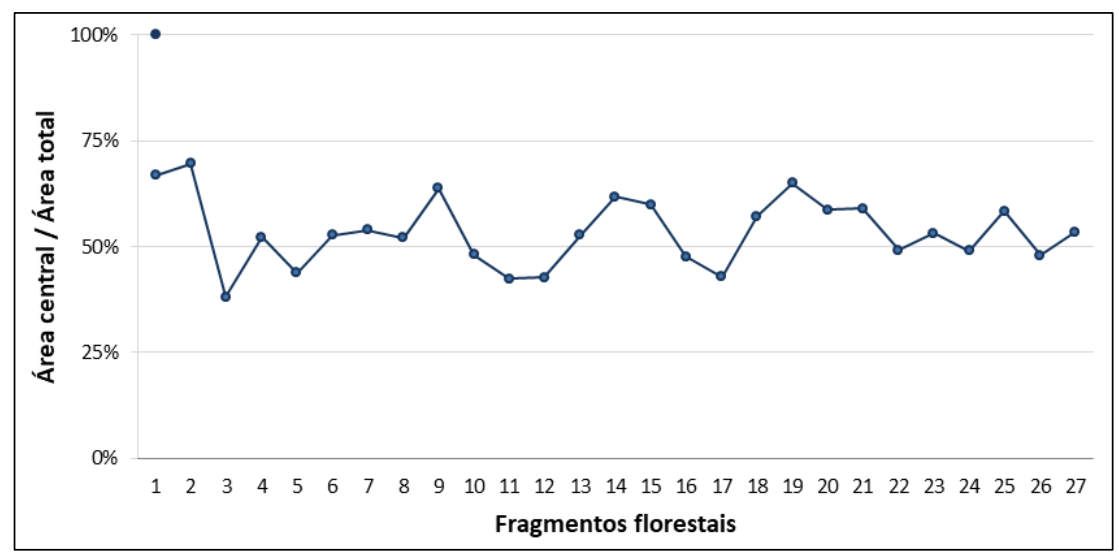

Figura 7: Relação área central / área total dos fragmentos.

Neste sentido, é possível afirmar que os fragmentos que apresentam um efeito de borda maior que sua área central estão mais susceptíveis ao processo de supressão da vegetação por atividades antrópicas, especialmente o fragmento 3, que apresenta em seu entorno atividades agrícolas classificadas como culturas temporárias. Além do fragmento 3, os fragmentos 11, 12 e 17 encontram-se na mesma situação e vulneráveis as ações antrópicas, visto o tipo de classe predominante no seu entorno que é de culturas temporárias e sua relação da área central pela área total do fragmento é menor que $50 \%$.

\section{2. Índice de Circularidade (IC)}

A Figura 8 apresenta o mapa do IC dos fragmentos presentes na área de estudo. Os valores de IC encontrados para os fragmentos florestais estudados variaram de 0,39 a 0,79 (respectivamente fragmentos 9 , 24 e 1). Não houve ocorrência de fragmento classificado como arredondado, sendo que 8 fragmentos foram classificados como alongados e 19 como muito alongados, o que representou $29,6 \%$ e $70,4 \%$ do total de fragmentos, respectivamente.



Figura 8: Mapa de Índices de Circularidade dos fragmentos florestais. 
Destaca-se que fragmentos com formas irregulares e alongadas dificultam a conservação da biodiversidade do local devido à maior propensão à fragmentação e ao efeito de borda, enquanto formas arredondadas tendem a apresentar maior área central, consequentemente menor efeito de borda (VIANA e PINHEIRO, 1998; LAURANCE e VASCONCELOS, 2009).

\section{3. Índice de Efeito de Borda (IEB)}

Os valores de IEB apresentaram amplitude de 0,32 , sendo que o menor valor encontrado foi 0,33 para o fragmento 12 na parte central da Figura 8 e o maior foi 0,65 para o fragmento 3 na parte nordeste da Figura 9. Dentre os fragmentos estudados, $5(18,5 \%)$ foram classificados como de alto impacto decorrente do uso e manejo do seu entorno e $22(81,5 \%)$ como de médio efeito, não sendo encontrado nenhum com baixo impacto (Figura 9).



Figura 9. Mapa de Índices de Efeito de Borda dos fragmentos florestais.

Os fragmentos com os piores cenários apresentam maior restrição à conservação devido ao tipo de uso da terra predominante, como áreas agrícolas, onde a intensa utilização antrópica inviabiliza a expansão e conservação da vegetação. Essa fragmentação e alterações nas bordas dos fragmentos podem promover alterações nas condições do local, afetando parâmetros físicos, químicos e biológicos, como a disponibilidade energética, fluxo de organismos entre os ambientes, incidência solar, umidade, vento nas bordas dos fragmentos, distribuição e abundância de espécies e interação entre organismos (VIANA; PINHEIRO, 1998; LOURENÇO; SILVA; SALES, 2014), especialmente para os fragmentos 1, 4, 6, 9 e 12 que se encontram altamente expostos às atividades antrópicas.

\section{4. Índice de Qualidade Biofísica do Fragmento (IQBF)}

Os valores médios de argila de cada fragmento variaram de 28,5\% (fragmento 9) a 55,2\% (fragmento 2). Maior presença de argila resulta em uma maior capacidade de retenção de água e, junto com a temperatura, auxilia a atividade biológica, influenciando no processo de degradação da matéria orgânica (LA ESCALA et al., 2000; DIAS, 2006; SIMONETTI et al., 2019).

Já o SAVI apresentou valores variando de 0,67 a 0,71 , sendo que o fragmento 10 obteve o menor valor e o fragmento 15 o maior. Como os índices de vegetação se utilizam das bandas do vermelho e infravermelho 
próximo e estas faixas representam a maior parte da resposta espectral da vegetação, estes índices manifestam a condição biofísica da vegetação, correlacionando com o vigor da vegetação verde, porcentagem de cobertura do solo, atividade fotossintética e produtividade (ROSA, 2009; LIMA et al., 2013).

$\mathrm{O}$ resultado de IQBF, obtido pela média da argila e do SAVI, variaram entre 0,49 (fragmentos 3 no nordeste e 9 no oeste) e 0,62 (fragmento 2 no norte). Do total de fragmentos, 2 foram classificados como de baixa, 23 como média e 2 como de alta qualidade biofísica, o que representou 7,4\%, 85,2\% e 7,4\%, respectivamente. A maior amplitude nos valores de argila fez com que este parâmetro tivesse maior influência no resultado do índice, isto é, com os maiores valores de argila expressando os maiores IQBF (Figura 10).

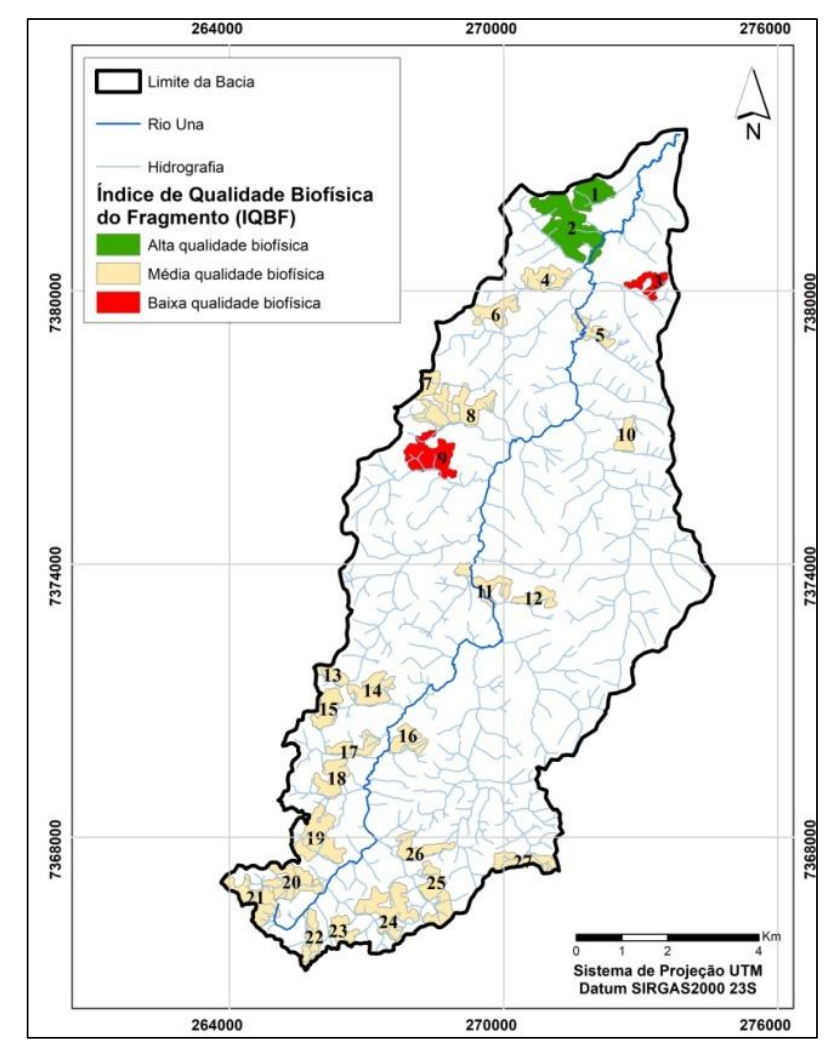

Figura 10. Mapa de Índices de Qualidade Biofísica dos fragmentos florestais.

Percebe-se que os fragmentos presentes nas áreas topográficas menos acidentadas (norte da bacia) apresentaram melhores respostas para o IQBF justamente por não sofrerem com a perda acentuada de solo devido a processos erosivos, conforme pode ser confirmado no estudo realizado por Silva et al. (2016) na bacia.

\subsection{Indicador de Fragilidade Florestal (IFF)}

Para o cálculo do IFF, foi construída uma matriz dos índices aos quais foram atribuídos pesos de importância mediante consulta a pesquisadores e posteriormente foi realizada a comparação pareada utilizando o método AHP (Tabela 2).

Tabela 2: Pesos atribuídos às categorias de uso e ocupação da terra

\begin{tabular}{llll}
\hline & IC & IEB & IQBF \\
\hline IC & 1 & $1 / 7$ & $1 / 4$ \\
IEB & 7 & 1 & 4 \\
IQBF & 4 & $1 / 4$ & 1 \\
\hline
\end{tabular}

Os pesos atribuídos foram de 0,09 para IC, 0,70 para o IEB e 0,21 para o IQBF. O índice de consistência (CI) da AHP foi 0,05 e a razão de consistência (RC) obtida foi de $8,8 \%$.

Os Indicadores de Fragilidade Florestal (IFF) encontrados mostraram apenas 1 fragmento classificado com IFF baixo, enquanto 22 foram classificados com IFF médio e 4 com IFF alto (Figura 11). 


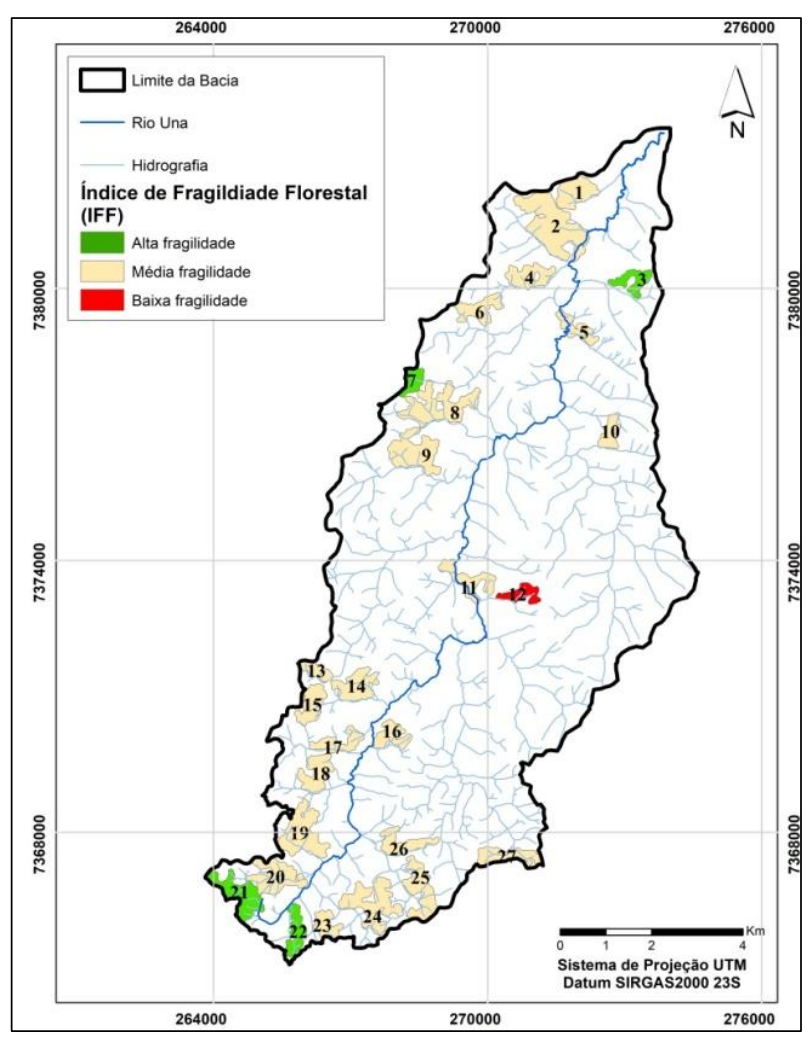

Figura 11: Mapa de Indicador de Fragilidade Florestal da Bacia Hidrográfica do Rio Una, SP, Brasil.

Os locais com baixo IFF apresentaram 3,7\% dos fragmentos estudados, totalizando uma área de 24,38 ha. Os fragmentos de médio IFF representaram a maioria dos fragmentos estudados, com $81,5 \%$ dos fragmentos e área total de 1.069,13ha. Já os fragmentos classificados com IFF alto ocorreram em 14,8\% e área total de 161,69 ha. Pode ser observado que o valor mínimo encontrado foi de 0,39 (fragmento 12 no centro) e o maior de 0,62 (fragmento 21 sul) (Tabela 3).

Tabela 3: Valores de IFF, ordenados do maior para o menor valor, dos fragmentos florestais da Bacia Hidrográfica do Rio Una, Ibiúna, SP.

\begin{tabular}{c|c|c|c|c|c}
\hline Ordem & Fragmento & IC & IEB & IQBF & IFF \\
\hline 1 & 21 & 0,54 & 0,64 & 0,56 & $\mathbf{0 , 6 2}$ \\
\hline 2 & 7 & 0,61 & 0,63 & 0,54 & $\mathbf{0 , 6 1}$ \\
\hline 3 & 3 & 0,52 & 0,65 & 0,49 & $\mathbf{0 , 6 0}$ \\
\hline 4 & 22 & 0,59 & 0,61 & 0,55 & $\mathbf{0 , 6 0}$ \\
\hline 5 & 18 & 0,53 & 0,61 & 0,55 & $\mathbf{0 , 5 9}$ \\
\hline 6 & 25 & 0,58 & 0,59 & 0,56 & $\mathbf{0 , 5 8}$ \\
\hline 7 & 27 & 0,58 & 0,58 & 0,52 & $\mathbf{0 , 5 7}$ \\
\hline 8 & 13 & 0,58 & 0,54 & 0,50 & $\mathbf{0 , 5 3}$ \\
\hline 9 & 5 & 0,56 & 0,52 & 0,59 & $\mathbf{0 , 5 3}$ \\
\hline 10 & 20 & 0,56 & 0,51 & 0,55 & $\mathbf{0 , 5 2}$ \\
\hline 11 & 8 & 0,77 & 0,48 & 0,53 & $\mathbf{0 , 5 1}$ \\
\hline 12 & 16 & 0,67 & 0,47 & 0,57 & $\mathbf{0 , 5 1}$ \\
\hline 13 & 2 & 0,51 & 0,47 & 0,62 & $\mathbf{0 , 5 0}$ \\
\hline 14 & 19 & 0,69 & 0,46 & 0,57 & $\mathbf{0 , 5 0}$ \\
\hline 15 & 23 & 0,69 & 0,45 & 0,54 & $\mathbf{0 , 4 9}$ \\
\hline 16 & 10 & 0,54 & 0,45 & 0,59 & $\mathbf{0 , 4 9}$ \\
\hline
\end{tabular}




\begin{tabular}{c|c|c|c|c|c}
\hline Ordem & Fragmento & IC & IEB & IQBF & IFF \\
\hline 17 & 17 & 0,53 & 0,44 & 0,55 & $\mathbf{0 , 4 7}$ \\
\hline 18 & 1 & 0,79 & 0,39 & 0,61 & $\mathbf{0 , 4 7}$ \\
\hline 19 & 24 & 0,39 & 0,45 & 0,56 & $\mathbf{0 , 4 7}$ \\
\hline 20 & 14 & 0,78 & 0,42 & 0,50 & $\mathbf{0 , 4 7}$ \\
\hline 21 & 15 & 0,64 & 0,43 & 0,52 & $\mathbf{0 , 4 6}$ \\
\hline 22 & 26 & 0,54 & 0,42 & 0,55 & $\mathbf{0 , 4 6}$ \\
\hline 23 & 4 & 0,58 & 0,36 & 0,59 & $\mathbf{0 , 4 3}$ \\
\hline 24 & 11 & 0,46 & 0,40 & 0,51 & $\mathbf{0 , 4 3}$ \\
\hline 25 & 6 & 0,58 & 0,35 & 0,57 & $\mathbf{0 , 4 2}$ \\
\hline 26 & 9 & 0,39 & 0,39 & 0,49 & $\mathbf{0 , 4 1}$ \\
\hline 27 & 12 & 0,59 & 0,33 & 0,51 & $\mathbf{0 , 3 9}$ \\
\hline
\end{tabular}

Os resultados do mapa da Figura 11 mostram que o maior valor de IFF encontrado foi resultante da combinação entre o $18^{\circ}$ maior valor de IC, $2^{\circ}$ valor de IEB e o $11^{\circ}$ valor de IQBF e o menor obteve o $9^{\circ}$ valor de IC, $27^{\circ}$ de IEB e $23^{\circ}$ de IQBF (Tabela 3 ). Isto reforça o fato de que devido ao peso do IEB ser quase 8 vezes maior que o IC e 3 vezes maior que o IQBF, o uso e manejo do entorno do fragmento contribuiu de forma mais relevante para o resultado obtido do que sua forma ou as condições biofísicas do local. Lourenço, Silva e Sales (2014) também concluíram em seu estudo sobre processo de fragmentação florestal no município de Sorocaba (SP) que a pressão antrópica é o principal fator impulsionador, sendo mais impactante para o fragmento quando este está exposto a atividades agrícolas conforme Sales et al. (2017) verificou em seus estudos sobre a abundância da avifauna presente nos fragmentos florestais na bacia hidrográfica do rio Una.

\section{Conclusões}

A elaboração de indicadores cartográficos é um importante instrumento no controle e tomada de decisão visando à gestão e o planejamento ambiental no âmbito da discussão de políticas públicas para a manutenção de importantes fragmentos florestais. Neste sentido, este indicador buscou avaliar as métricas dos fragmentos florestais, condição do solo, exposição às atividades antrópicas e condição de saudabilidade da vegetação por meio da interação da vegetação com a radiação eletromagnética, possibilitando, desta forma, ao gestor avaliar um alto volume de informações impossíveis de serem analisadas de forma tabular.

O Indicador de Fragilidade Florestal (IFF) permitiu classificar a atual situação de fragilidade dos fragmentos florestais e identificar quais fragmentos carecem de maior atenção para sua manutenção e conservação, sendo identificado 3 fragmentos ao longo da bacia como de alta fragilidade.

A partir dos resultados do IFF, percebe-se que o tipo de classe de uso da terra no entorno é o principal fator impulsionador do processo de fragmentação, sendo mais impactante para o fragmento quando este está exposto a atividades agrícolas, o que corrobora com a paisagem degradada da bacia que apresenta aproximadamente $60 \%$ do seu território ocupado por atividades antrópicas, portanto, o método foi capaz de mostrar que o tipo de uso e manejo da terra nas áreas adjacentes aos fragmentos florestais são os principais fatores de desencadeamento de processos de degradação dos remanescentes florestais.

\section{Referências Bibliográficas}

AMORIM, A. T.; SOUSA, J. A. P.; LOURENÇO, R. W. Indicador dos estágios de sucessão de fragmentos florestais do bioma Mata Atlântica. Rev. Bras. Cartogr., vol. 71, n. 3, pp. 756 - 780, 2019. https://doi.org/10.14393/rbcv71n3-48546

BITTAR, I. M. B.; FERREIRA, A. S.; CÔRREA, G. F. Influência da textura do solo na atividade microbiana, decomposição e mineralização do carbono de serapilheira de sítios do bioma cerrado sob condições de incubação. Bioscience Journal, v. 29, n. 6, p. 1952-1960, 2013. 
BORGES, L. F. R., SCOLFORO, J. P., OLIVEIRA, A. D., MELlO, J. D, ACERBI JUNIOR, F. W.; FREITAS, G. D. Inventário de fragmentos florestais nativos e propostas para seu manejo e o da paisagem. Cerne, 10(1): 22-38, 2004. Disponível em: https://www.redalyc.org/articulo.oa?id=744/74410103

BURROUGH, P. Principles of geography information systems for land resources assessment. Oxford: Oxford University Press, 333p. 1998.

COSTA, T. C.; BELDERRAIN, M. C. N. Decisão em grupo em métodos multicritério de apoio à decisão. Anais do $1^{\circ}$ Encontro de Iniciação Científica e Pós-Graduação do ITA XV ENCITA. Instituto Tecnológico de Aeronáutica, São José dos Campos, SP, Brasil, 2009.

DIAS, J. D. Fluxo de $\mathrm{CO}_{2}$ proveniente da respiração do solo em áreas de floresta nativa da Amazônia. 2006. 88p. Dissertação (Mestrado em Agronomia) - Escola Superior de Agronomia Luiz de Queiroz, Piracicaba, 2006. https://doi.org/10.11606/D.91.2006.tde-04102006-163445

DRANSKI, A. Espacialização de atributos químicos e do teor de argila e suas relações com as curvas espectrais em área de latossolo bruno. Dissertação (Mestrado em Agronomia) - Universidade Estadual do Centro-Oeste. 2016.

FAHRIG, L. Effects of Habitat Fragmentation on Biodiversity. Annual Review of Ecology, Evolution, and Systematics, v. 34, n. 1, p. 487-515, 2003. https://doi.org/10.1146/annurev.ecolsys.34.011802.132419

GOMES, K. G. A. Um método multicritério para localização de unidades celulares de intendência da FAB. Dissertação (Mestrado em Logística) - Departamento de Engenharia de Produção, Pontifícia Universidade Católica do Rio de Janeiro, Rio de Janeiro. 2009.

HANSKI, I.; GILPIN, M. E. Metapopulation Biology: Ecology, Genetics and Evolution. Academic Press, San Diego, CA, 1997.

HUETE, A. R. A soil-adjusted vegetation index (SAVI). Remote Sensing of Environment. v. 25, p. 295309, 1988. https://doi.org/10.1016/0034-4257(88)90106-X

IBGE. Instituto Brasileiro de Geografia e Estatística. Manual técnico de uso da terra. $3^{\text {a }}$ Ed. Rio de Janeiro: IBGE. 171p. 2013a.

IBGE. Instituto Brasileiro de Geografia e Estatística. Produção Agrícola Municipal. Culturas Temporárias e permanentes. v. 40, 2013b. 102p.

IBIÚNA. Dados da cidade. Disponível em http://www.ibiuna.sp.gov.br/menu/a-cidade. Acesso em 08 mai. 2018.

KRONKA et al. Inventário florestal da vegetação natural do Estado de São Paulo. São Paulo: Secretaria do Meio Ambiente; Instituto Florestal, 2005. 200 p.

LA ESCALA Jr., N.; MARQUES J. R.; PEREIRA, J. T.; CORA, J. E. Short-term temporal changes in the spatial variability model of $\mathrm{CO}_{2}$ emissions from Brazilian bare soil. Soil biology \& biochemistry, v. 32, p. 1459-1462, 2000. https://doi.org/10.1016/S0038-0717(00)00051-1

LAURANCE, W. F. Conserving the hottest of the hotspots. Biol. Conserv. 142,1137, 2009. https://doi.org/10.1016/j.biocon.2008.10.011

LAURANCE, W. F.; VASCONCELOS, H. L. Consequências ecológicas da fragmentação florestal na Amazônia. Oecologia brasiliensis, v. 13, n. $03, \quad$ p. $434-451, \quad$ set. 2009. http://dx.doi.org/10.4257/oeco.2009.1303.03

LIMA, G. C.; SILVA, M. L. N.; CURI, N.; SILVA, M. A.; OLIVEIRA, A. H.; AVANZI, J. C.; UMMUS, M. E. Avaliação da cobertura vegetal pelo índice de vegetação por diferença normalizada (IVDN). AmbiAgua, Taubaté, v. 8, n. 2, p. 204-214, 2013. http://dx.doi.org/10.4136/ambi-agua.959 
LOURENÇO, R. W.; SIlVA, D. C. C.; MARTINS, A. C. G.; SAlES, J. C. A.; ROVEDA, S. R. M. M.; ROVEDA, J. A. F. Use of fuzzy systems in the elaboration of an anthropic pressure indicator to evaluate the remaining forest fragments. Environmental Earth Sciences, v. $73, \quad$ p. $1-8, \quad 2015$. https://doi.org/10.1007/s12665-015-4253-6

LOURENÇO, R. W.; SILVA, D. C. C.; SALES, J. C. A. Elaboração de uma metodologia de avaliação de fragmentos de remanescentes florestais como ferramenta de gestão e planejamento ambiental. Ambiência, v.10, n.3 p. 685 - 698, 2014. https://doi.org/10.5935/ambiencia.2014.03.03

OliveIRA, L. S. C.; MARANGON, L. C.; FEliCiAnO, A. L. P.; LiMA, A. S.; CARDOSO, M. S. O.; DOS SANTOS, W. B. Efeito de borda em remanescentes de floresta atlântica na bacia do rio Tapacurá, Pernambuco. Cerne, v.21, n.2, p.169-174, 2015. https://doi.org/10.1590 / 01047760201521021185

OSTOVARI, Y.; HONARBAKHSH, A.; SANGOONY, H.; ZOLFAGHARI, F.; MALEKI, K. \& INGRAM, B. GIS and multi-criteria decision-making analysis assessment of land suitability for rapeseed farming in calcareous soils of semi-arid regions, Ecological Indicators, 103, 479-487, 2019.

https://doi.org/10.1016/j.ecolind.2019.04.051

PONZONI, F.J.; SHIMABUKURO, Y.E. Sensoriamento remoto aplicado ao estudo da vegetação. 1 ed. São José dos Campos: Parêntese, 135 p. 2007.

RIBEIRO, M. C.; METZGER, J. P.; MARTENSEN, A. C.; PONZONI, F. J.; HIROTA M. M. The Brazilian Atlantic Forest: How much is left, and how is the remaining forest distributed? Implications for conservation. Biological Conservation (142), p.1144-1156, 2009. https://doi.org/10.1016/j.biocon.2009.02.021

ROSA, R. Introdução ao Sensoriamento Remoto. $7^{a}$ ed., Uberlândia: EDUFU, 264 p. 2009.

SAATY, T. Método de análise hierárquica. São Paulo: McGraw-Hill. 1991.

SALES, J. C. A.; SILVA, SILVA, D.C.C.; BITAR, O. Y.; LOURENÇO, R. W. Proposal of methodology for spatial analysis applied to human development index in water basins. GEOJOURNAL, v. 84, p. 813-828, 2019. https://doi.org/10.1007/s10708-018-9894-z

SALES, J. C. A.; SILVA, D. C. C.; LOURENÇO, R. W. Identificação de áreas prioritárias para conservação da avifauna na bacia hidrográfica do rio Una, Ibiuna/SP. Revista Ibero-americana de Ciências Ambientais, v. 8, p. 128-142, 2017. https://doi.org/10.6008/SPC2179-6858.2017.004.0011

SANTOS, A. R.; PEluZIO, J. B. E.; PEluZIO, T. M. O.; SANTOS, G. M. A. D. A. S. Org. Geotecnologias aplicadas aos recursos florestais [recurso eletrônico] - Alegre, ES: CAUFES, 249 p. 2012.

SANTOS, M. S.; AMARAL, M.; SOUZA, J. C. Avaliação sazonal da cobertura vegetal da microrregião da Chapada dos Veadeiros por meio dos índices SAVI e NDWI. Élisée - Revista de Geografia da UEG, v. 8, p. 01-18, 2019.

SIlVA, D. C. C.; AlBuQuERQuE FIlHO, J. L.; OLIVEIRA, R. A.; LOURENÇO, R. W. Proposta Metodológica para Análise da Inserção Social na Bacia Hidrográfica do Rio Una em Ibiúna/SP. Revista do departamento de geografia, v. 33, p. 74-84, 2017. https://doi.org/10.11606/rdg.v33i0.125677

SILVA, D.C.C.; ALBUQUERQUE FILHO, J.L.; SALES, J.C.A.; LOURENÇO, R.W. Uso de indicadores morfométricos como ferramentas para avaliação de bacias hidrográficas. Revista Brasileira de Geografia Física, v. 9, p. 627-642, 2016. https://doi.org/10.26848/rbgf.v9.2.p627-642

SILVA, E. R. A. C.; MELO, J. G. S.; ASSIS, D. R. S.; SANTANA, S. H. C.; GANVÍNCIO, S. D. O desafio da gestão ambiental de florestas urbanas: análise da degradação ambiental da Reserva de Floresta Urbana Mata do Janga - PE através de técnicas de sensoriamento remoto. R. gest. sust. ambient., Florianópolis, v. 7 , n. 3, p.454-469, 2018. http://dx.doi.org/10.19177/rgsa.v7e32018454-469

SIMONETTI, V. C.; SABONARO, D. Z.; LOURENÇO, R. W.; ROSA, A. H.; SILVA, D. C. C. Análise da Variabilidade Espacial Horizontal e Vertical dos Atributos do Solo e sua Relevância para o Parque Natural 
Chico Mendes, SP. Revista Brasileira de Geografia Física, v. 12, p. 2537-2550, 2019. http://dx.doi.org/10.26848/rbgf.v12.7.p2537-2550

SOUZA, J. C.; LOPES, E. R. N.; SOUSA, J. A. P.; LOURENÇO, R. W. Avaliação dos aspectos físicoquímicos dos solos em diferentes coberturas vegetais. Revista Brasileira de Ciências Ambientais (Online), n. 49, p. 123-139, 2018. http://dx.doi.org/10.5327/Z2176-947820180366

SOUZA, J. C.; LOPES, E. R. N.; SOUSA, J. A. P.; PADOVANNI, N. G.; LOURENÇO, R. W. Dinâmica Espacial e Sazonal da Temperatura, Umidade e Estresse Hídrico em Diferentes Tipos de Cobertura Vegetal. Revista do Departamento de Geografia, v. 37, p. 80-94, 2019. https://doi.org/10.11606/rdg.v37i0.144716

TRINDADE, F. S.; ALVES, M. C.; NOETZOLD, R.; ANDRADE, I. C.; POZZA, A. A. A. Relação espectro-temporal de índices de vegetação com atributos do solo e produtividade da soja. Amazonian journal of agricultural and environmental sciences / Revista de ciências agrárias, v. 62, p. 1-11, 2019. http://dx.doi.org/10.22491/rca.2019.2928

VIANA, V. M.; PINHEIRO, L. A. F. V. Conservação da biodiversidade em fragmentos florestais. Série Técnica IPEF, Piracicaba, v. 12, n. 32, p. 25-42, 1998.

YIN, R. K. Estudo de caso: planejamento e métodos. Porto Alegre: Bookman, 2010.



